“( 2015 IEEE. Personal use of this material is permitted. Permission from IEEE must be obtained for all other uses, in any current or future media, including reprinting/republishing this material for advertising or promotional purposes, creating new collective works, for resale or redistribution to servers or lists, or reuse of any copyrighted component of this work in other works." 


\title{
Adaptive Searching and Tracking Algorithm for AoA Estimation in Localized Hybrid Array
}

\author{
Jian A. Zhang ${ }^{1}$, Xiaojing Huang ${ }^{2}$ and Y. Jay Guo ${ }^{2}$ \\ ${ }^{1}$ Wireless and Networking Program, Digital Productivity, CSIRO, Australia \\ ${ }^{2}$ University of Technology, Sydney \\ Email: Andrew.Zhang@csiro.au; \{Xiaojing.Huang; Jay.Guo\}@uts.edu.au
}

\begin{abstract}
Angle-of-Arrival estimation in localized hybrid array is very challenging due to the phase ambiguity problem. A differential beam searching (DBS) algorithm was proposed to solve the problem. However, it converges slowly and suffers from a zigzag effect where estimates may jump between correct and wrong estimates. In this paper, we first propose an improved DBS algorithm to mitigate the zigzag effect. We then analyze the error sources for general DBS algorithms, and propose a new adaptive searching and tracking (AST) algorithm. By using carefully designed updating functions and exit conditions, the proposed AST algorithm allows automatic switching between searching and tracking modes, and can significantly speed up estimation process and reduce estimation error. Numerical results are provided to verify the effectiveness of the proposed schemes.
\end{abstract}

\section{INTRODUCTION}

Millimeter wave (mm-wave) radio has been considered as a very promising candidate for $5 \mathrm{G}$ cellular communications thanks to the large available bandwidth [1]. To combat large propagation attenuation and support mobile users, mm-wave radio needs to use a steerable directional antenna array with many antenna elements. A full digital array, i.e., using a radio frequency (RF) front-end and digital baseband for each antenna, can be very costly in this case. A hybrid array [2], [3], which consists of multiple analog subarrays with each subarray having its digital chain, turns out to be a more feasible solution. It can not only provide a significant saving on cost and complexity, but also achieve comparable performance thanks to the multipath sparsity [1], [4]. There are typically two types of hybrid arrays, localized and interleaved arrays. In a localized array, antenna elements belonging to each analog subarray are adjacent to each other; while in an interleaved array, they scatter uniformly over the whole array.

Angle-of-arrival (AoA) estimation in a mm-wave hybrid array is a very important problem and forms the basis for many advanced processing techniques such as beamforming, single user multiple input multiple output (MIMO), and spatial division multiuser access [4]. AoA estimation in a hybrid array is quite different from those well-studied ones in either a full analog or digital array. A full analog array generally uses beam scanning to search the AoA [5], while a full digital array can estimate it in one step using, e.g., spectrum analysis techniques and maximal likelihood estimator [6]. For a hybrid array, existing algorithms need to be adapted to the special architecture and to different subarray configurations. The AoA estimation algorithms for a hybrid array typically need to be implemented recursively between digital and analog parts.

Due to the multipath sparsity, typical mm-wave channels have strong line-of-sight (LOS) component and much weaker non-LOS ones. Here we are interested in estimating the LOS component only. For estimating the AoA of a single incoming signal, one technique is to exploit the constant phase difference between corresponding elements in two neigbouring subarrays, as proposed in [7], where a differential beam tracking (DBT) algorithm and a differential beam search (DBS) algorithm are proposed for interleaved and localized arrays, respectively. The need for two different algorithms is mainly due to the phase ambiguity problem in the localized configuration. DBS can remove the phase ambiguity, at the cost of increased complexity and significantly reduced convergence speed. Two special problems to be discussed later, phase discontinuity and error in the intermediate estimate, can also cause a zigzag effect in the final estimate, where the estimate jumps between correct and wrong values. To avoid the searching process in DBS and solve the phase ambiguity problem, a frequency-domain AoA algorithm is proposed in [8]. Unfortunately, this approach relies on the product of two cross-correlation values and has significantly higher noise than the time domain approach. A codebook searching based scheme is also proposed in [9].

In this paper, we investigate better algorithms for AoA estimation in localized hybrid arrays. We first propose an improved DBS algorithm, analyze the limitations of this type of algorithm, and then propose a significantly better adaptive searching and tracking (AST) algorithm. Via carefully designed updating functions and exit conditions, the AST algorithm can automatically switch between searching and tracking modes. This can effectively solve the two special problems in conventional DBS algorithms, and enables a much faster estimation process.

\section{Problem Formulation}

We consider a localized square hybrid array which consists of $M=M_{x} \times M_{y}$ square subarrays. Each square subarray is an analog array, consisting of $N=N_{x} \times N_{y}$ adjacent antennas connected with analogue adjustable phase shifters in the RF chain. Each subarray is connected to a baseband processor via a digital-to-analog convertor (DAC) in the transmitter or an analog-to-digital convertor (ADC) in the receiver. The distance between all adjacent antennas is assumed to be the same and is denoted as $d$. 
Assume that each antenna has an omni-directional radiation pattern, i.e., spatial response 1 over all angles. For incoming signals $\tilde{s}(t)$ with wavelength $\lambda_{c}$ at elevation angle $\theta$ and azimuth angle $\phi$, the received signal at the $\left(m_{x}, m_{y}\right)$-th subarray can be represented as [7]

$$
\begin{aligned}
& s_{m_{x}, m_{y}}(t) \\
& =\tilde{s}(t) P_{m_{x}, m_{y}}(\theta, \phi) e^{j\left(m_{x} N_{x} u_{x}+m_{y} N_{y} u_{y}\right)}+\xi_{m_{x}, m_{y}}(t),
\end{aligned}
$$

where $\xi_{m_{x}, m_{y}}(t)$ is the sum of all the AWGN noise from $N_{x} N_{y}$ antenna elements, and

$$
\begin{aligned}
& u_{x}=2 \pi d \sin (\theta) \cos (\phi) / \lambda_{c} \\
& u_{y}=2 \pi d \sin (\theta) \sin (\phi) / \lambda_{c} \\
& P_{m_{x}, m_{y}}(\theta, \phi)=\sum_{i_{x}=0}^{N_{x}-1} \sum_{i_{y}=0}^{N_{y}-1} e^{j\left(i_{x} u_{x}+i_{y} u_{y}+\alpha_{m_{x}, m_{y}}\left(i_{x}, i_{y}\right)\right)}
\end{aligned}
$$

$\alpha_{m_{x}, m_{y}}\left(i_{x}, i_{y}\right)$ is the phase shift value at the $\left(i_{x}, i_{y}\right)$-th antenna in the $\left(m_{x}, m_{y}\right)$-th subarray, and $j=\sqrt{-1}$.

Now the task for the AOA estimation is to determine the values of $u_{x}$ and $u_{y}$ from the observation signals $s_{m_{x}, m_{y}}(t)$.

When $\alpha_{m_{x}, m_{y}}\left(i_{x}, i_{y}\right)=\alpha\left(i_{x}, i_{y}\right)$ is chosen as the same for the $\left(i_{x}, i_{y}\right)$-th antenna in all subarrays, $P(\theta, \phi) \triangleq$ $P_{m_{x}, m_{y}}(\theta, \phi)$, and the cross-correlation between any two neighbouring subarrays along $\mathrm{x}$-axis or $\mathrm{y}$-axis is given by [7]

$$
\begin{aligned}
& R_{x}=E\left\{|\tilde{s}(t)|^{2}\right\}|P(\theta, \Phi)|^{2} e^{j N_{x} u_{x}}, \text { or } \\
& R_{y}=E\left\{|\tilde{s}(t)|^{2}\right\}|P(\theta, \Phi)|^{2} e^{j N_{y} u_{y}} .
\end{aligned}
$$

Hence the AoA parameters $u_{x}$ and $u_{y}$ can be estimated via $\arg \left\{R_{x}\right\}$ and $\arg \left\{R_{y}\right\}$, the angle of $R_{x}$ and $R_{y}$, respectively. However, a phase ambiguity problem arises as both $u_{x}$ and $u_{y}$ can take values beyond $[-\pi, \pi]$ while $\arg \{\cdot\} \in[-\pi, \pi)$. For convenience, we will use $u$ to represent either $u_{x}$ or $u_{y}$, and similarly for other symbols, if no identification is needed.

Consider the case of $d=\lambda_{c} / 2$. We have $u \in[-\pi, \pi]$ for $\theta, \phi \in[-\pi, \pi]$. The actual value of $u$ could be one out of $N$, in the form of

$$
\begin{aligned}
& u_{x}\left(q_{x}\right)=\bmod \left(\left(\arg \left\{R_{x}\right\}+2 \pi q_{x}\right) / N_{x}, 2 \pi\right)-\pi, \\
& u_{y}\left(q_{y}\right)=\bmod \left(\left(\arg \left\{R_{y}\right\}+2 \pi q_{y}\right) / N_{y}, 2 \pi\right)-\pi,
\end{aligned}
$$

where both $q_{x}$ and $q_{y}$ are integers, and $q_{x}, q_{y} \in\left[0, N_{x}-1\right]$.

Determining the right $\hat{q}_{x}$ and $\hat{q}_{y}$ such that $u_{x}\left(\hat{q}_{x}\right)=u_{x}$ and $u_{y}\left(\hat{q}_{x}\right)=u_{y}$ is a challenging problem due to the discontinuity of the $\arg \{\cdot\}$ function at $\pi$ and $-\pi$ and the estimation error in $\arg \{\cdot\}$, as will be shown in Section III.

\section{DBS ALGORITHMS AND THEIR LIMITATIONS}

To solve this phase ambiguity problem, a DBS algorithm is proposed in [7]. In the algorithm, the phase ambiguity is solved by independently testing every combination of $q_{x}$ and $q_{y}$. For each pair of $\left(q_{x}, q_{y}\right)$, a subframe is allocated within one frame (iteration) and the estimation of its $u_{x}\left(q_{x}\right)$ and $u_{y}\left(q_{y}\right)$ are conducted using the approach as described Section II. The process is repeated over multiple frames with updated subarray

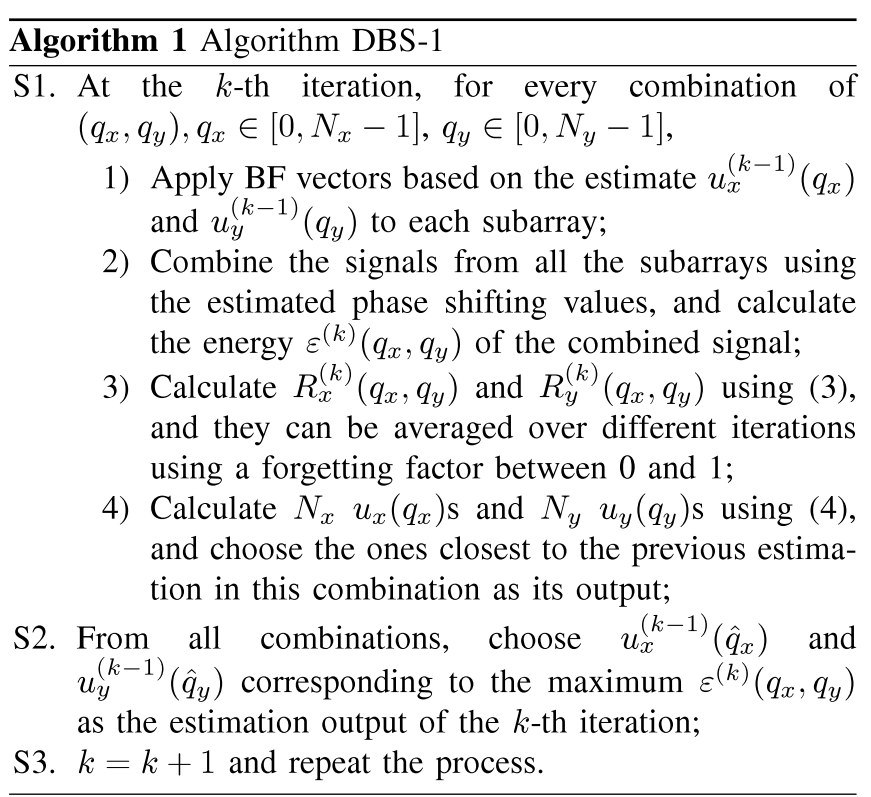

phase shifting values generated from $u_{x}\left(q_{x}\right)$ and $u_{y}\left(q_{y}\right)$. The signal from all the subarrays are collected and combined as

$s(t)=$
$\sum_{m_{x}, m_{y}}\left(s_{m_{x}, m_{y}}(t) e^{-j\left(m_{x} \arg \left\{R_{x}\right\}+m_{y} \arg \left\{R_{y}\right\}\right)}+\xi_{m_{x} m_{y}}(t)\right)$.

The energy of the combined signal is compared, and the final estimates $u_{x}\left(\hat{q}_{x}\right)$ and $u_{y}\left(\hat{q}_{x}\right)$ are obtained as those in the combination with the maximum energy in each iteration.

Using the new estimates $u_{x}\left(\hat{q}_{x}\right)$ and $u_{y}\left(\hat{q}_{x}\right)$ as the estimation output in the current iteration can be problematic, as the maximum power corresponds to $u$ in the previous estimation. In other words, the new estimates are yet to be tested whether they can generate the maximum energy in the next iteration. Here we propose a slightly improved DBS algorithm, as summarized in Algorithm 1 and denoted as DBS-1, trying to fix this problem.

Compared to DBS, the main change is as follows: For each combination of $\left(q_{x}, q_{y}\right)$, we calculate $N$ possible values for $u$ after obtaining the new estimates $\arg \{R\}$, and then choose the one closest to the previous estimate $u$ for this combination. From all the combinations, we then choose the one corresponding to the maximum energy as the estimation output in this iteration. Another change is that the searching space is reduced from $\left(N_{x}+1\right)\left(N_{y}+1\right)$ to $N_{x} N_{y}$. This is due to the fact that $u_{x}\left(q_{x}\right)$ and $u_{y}\left(q_{y}\right)$ are periodic functions with a period of $N_{x}$ and $N_{y}$ as can be seen from (4).

Note that in Algorithm DBS-1, each combination only uses its own estimate in the previous iteration as an input into the current iteration. This is helpful for preventing error propagation, but also uses a lot of signals in scanning wrong AoAs.

Similar to the original DBS algorithm, DBS-1 works well for small AoA values and when SNR is not very low. However, both of them suffer from convergence problems, primarily due to the phase discontinuity problem and the estimation error in $\arg \{R\}$. These problems cause the "zigzag" effect, where 


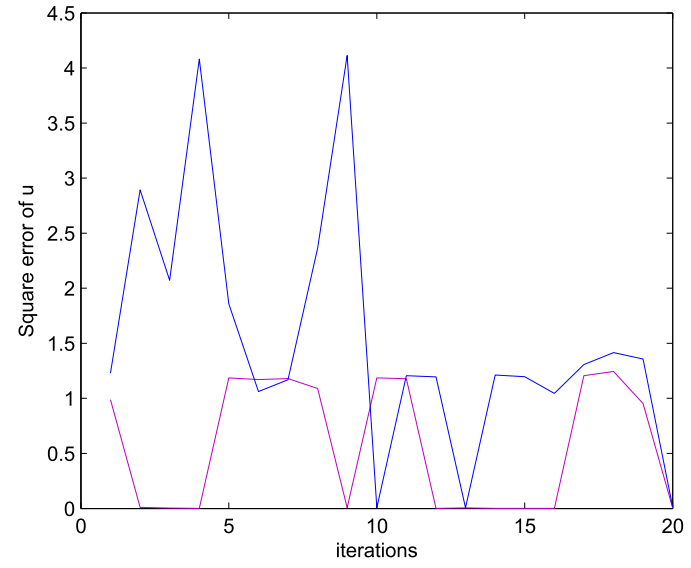

Fig. 1. The zigzag effect in DBS algorithms, where two random trials are shown.

the estimates jump between correct and wrong values, as can be observed from Fig. 1, which shows the square error of estimates for 2 trials using DBS-1.

\section{A. Estimation Error in $\arg \{R\}$ and its Impact}

In each iteration, the phase shifting value used in the $\left(i_{x}, i_{y}\right)$-th subarray, determined from the estimates $\arg \left\{R_{x}\right\}$ and $\arg \left\{R_{y}\right\}$, are given by

$$
\alpha\left(i_{x}, i_{y}\right)=-\left(i_{x} u_{x}+i_{y} u_{y}\right) .
$$

Let the estimation errors in $\arg \left\{R_{x}\right\}$ and $\arg \left\{R_{y}\right\}$ be $\delta_{x}$ and $\delta_{y}$, respectively. Let $\hat{q}_{x}$ and $\hat{q}_{y}$ denote the actual integers that generate $u_{x}$ and $u_{y}$ in (4). After applying the phase shifting values with any $q_{x}$ and $q_{y}$, the overall radiation pattern of a subarray is obtained as

$$
\begin{aligned}
& P\left(\theta, \phi, q_{x}, q_{y}\right) \\
& =\sum_{i_{x}=0}^{N_{x}-1} \sum_{i_{y}=0}^{N_{y}-1} e^{j\left(\left(\delta_{x}+2 \pi\left(q_{x}-\hat{q}_{x}\right)\right) i_{x} / N_{x}+\left(\delta_{y}+2 \pi\left(q_{y}-\hat{q}_{y}\right) i_{y} / N_{y}\right)\right.} \\
& =\frac{\left(1-e^{j \delta_{x}}\right)\left(1-e^{j \delta_{y}}\right)}{\left(1-e^{j\left(\delta_{x}+2 \pi\left(q_{x}-\hat{q}_{x}\right)\right) / N_{x}}\right)\left(1-e^{\left.j\left(\delta_{y}+2 \pi\left(q_{y}-\hat{q}_{y}\right)\right) / N_{y}\right)}\right.}
\end{aligned}
$$

when the denominator is not zero. The denominator will be zero in the cases of either $e^{-j\left(\delta_{x}+2 \pi\left(q_{x}-\hat{q}_{x}\right)\right) / N_{x}}=1$ or $e^{-j\left(\delta_{y}+2 \pi\left(q_{y}-\hat{q}_{y}\right)\right) / N_{y}}=1$. Given $\delta_{x}, \delta_{y} \in[-\pi, \pi]$ and $q_{x}-\hat{q}_{x} \in\left[-N_{x}+1, N_{x}-1\right], q_{y}-\hat{q}_{y} \in\left[-N_{y}+1, N_{y}-1\right]$, this could only happen when $\delta_{x}=0, q_{x}=\hat{q}_{x}$ or $\delta_{y}=0, q_{y}=\hat{q}_{y}$ In this case,

$$
\begin{aligned}
& P\left(\theta, \phi, q_{x}, q_{y}\right) \\
& =\left\{\begin{array}{cc}
\frac{N_{x}\left(1-e^{j \delta_{y}}\right)}{\left(1-e^{\left.j\left(\delta_{y}+2 \pi\left(q_{y}-\hat{q}_{y}\right)\right) / N_{y}\right)},\right.} & \text { when } \delta_{x}=0, q_{x}=\hat{q}_{x}, \text { only } \\
\frac{N_{y}\left(1-e^{j \delta_{x}}\right)}{\left(1-e^{\left.j\left(\delta_{x}+2 \pi\left(q_{x}-\hat{q}_{x}\right)\right) / N_{x}\right)}\right.}, & \text { when } \delta_{y}=0, q_{y}=\hat{q}_{y}, \text { only } \\
N_{x} N_{y}, & \text { when both are true. }
\end{array}\right.
\end{aligned}
$$

The numerator and $P\left(\theta, \phi, q_{x}, q_{y}\right)$ in (7) become 0 , if $\delta_{x}=$ $0, q_{x} \neq \hat{q}_{x}$ or $\delta_{y}=0, q_{y} \neq \hat{q}_{y}$.

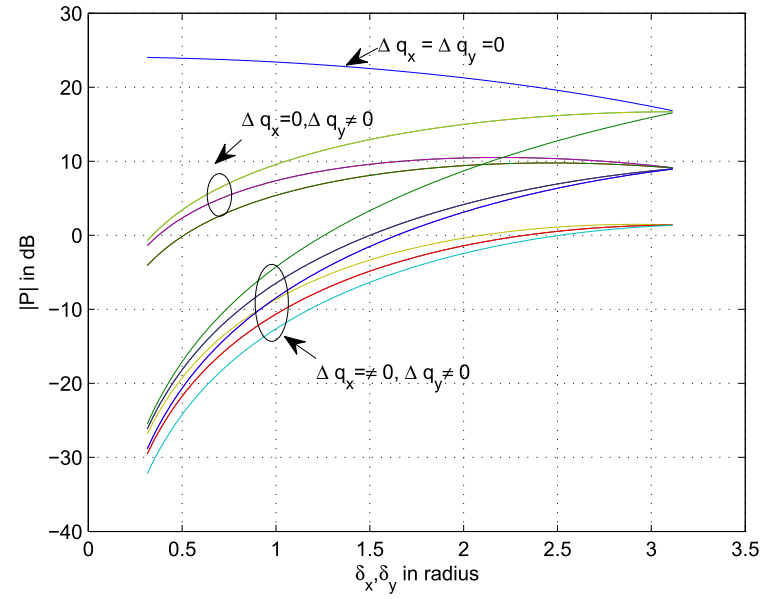

Fig. 2. Variation of $\left|P\left(\theta, \phi, q_{x}, q_{y}\right)\right|$ (in $\left.\mathrm{dB}\right)$ with the estimation error $\delta_{x}=$ $\delta_{y}$ and different values of $\Delta q=q-\hat{q}$.

In Fig. 2, we demonstrate how $\left|P\left(\theta, \phi, q_{x}, q_{y}\right)\right|$ changes with different $\Delta q=q-\hat{q}$ at various estimation errors $\delta_{x}=\delta_{y}$, when $N_{x}=N_{y}=4$. It can be seen that the gap between $|P|$ for $\Delta q_{x}=\Delta q_{y}=0$ and the other cases decreases quickly with $\delta$ increasing. The gap at small $\delta$ is large enough such that the right $q_{x}$ can be found even at very low SNRs. However, at larger $\delta$, such as $\delta>0.8$, the gap becomes less than $15 \mathrm{~dB}$. A wrong $q$ may be chosen at a low SNR when this gap is filled by noise. This can cause the difficulty in getting a good initial estimate for $\arg \{R\}$, and it may also lead the iteration to the wrong direction. Therefore, the capability of an algorithm in reducing $\delta$ is critical, particularly at a low SNR.

\section{B. Phase Discontinuity Problem}

Phase discontinuity is a special but neglected problem in AoA estimation in a localized hybrid array. The value of $\arg \{R\}$ is in the range of $[-\pi, \pi]$, and the phase discontinuity problem arises when the actual value of $|\arg \{R\}|$ is close to $\pi$. In the presence of noise, for example, an actual $\arg \{R\}=0.95 \pi$ may be estimated as $-0.98 \pi$. Although these two values are close in angle, they lead to significantly different $\exp (j \arg \{R\} / N)$, and then $u$ for a given $q$. This can cause $u(q)$ for a given $q$ to jump between different values and lead to non-convergence and the zigzag effect.

\section{AdAPtive SeARChing AND TRACKIng (AST) ALGORITHM}

The basic idea here is to get a good enough initial estimate via scanning/searching, and then use this estimate as an anchor point to determine $u$ from $\arg \{R\}$ without causing the miszoning problem in the following iterations. Once such an anchor point is obtained, we also want to end searching and move to another mode to shorten the estimation period. A mechanism is designed to enable the algorithm to go back to the searching mode if the anchor point is incorrectly chosen. Next, we present the algorithm and discuss its design philosophy. 


\section{A. AST Algorithm and Design Philosophy}

The proposed AST algorithm includes two interchangeable stages, searching and tracking stages. In the searching stage, AST tries to find a good initial estimate for $\arg \{R\}$ as smaller $\delta$ can lead to better correctness in determining $q$. This requires larger received signal energy through analog beamforming. To increase received signal energy, AST will scan refined angles until the energy of the received signal satisfies a condition, named as Condition A. Once condition A is met and an initial estimate is obtained, AST will move to the tracking stage, where iterations continue without searching. The energy of the received signal will also be monitored in the tracking stage. If it violates another condition, called as Condition $\mathrm{B}$, then the algorithm moves back to the searching stage. Details of the algorithm are described in Algorithm 2 and 3.

The way of using previous estimate in the current iteration is important. On one hand, we want to exploit previous estimate in the current iteration. On the other hand, we would like to allow a refined/changed searching angle if Condition $\mathrm{A}$ is not satisfied in the searching stage, and allow an expanded searching if condition B is not satisfied in the tracking stage. To achieve this goal, we propose the following updating functions:

In the $k$-th iteration, signals from $N_{x} N_{y}$ antennas in every subarray are combined using analog BF vectors based on

$$
\begin{aligned}
& w_{x}^{(k)}\left(q_{x}\right)= \\
& \left\{\begin{array}{cc}
u_{x}^{(k-1)}\left(\hat{q}_{x}\right), & q_{x}=\hat{q}_{x} ; \\
u_{x}^{(k-1)}\left(\hat{q}_{x}\right)+\alpha^{(k)}+2 \pi q_{x} / N_{x}, & q_{x} \neq \hat{q}_{x} ;
\end{array}\right.
\end{aligned}
$$

and

$$
\begin{aligned}
& w_{y}^{(k)}\left(q_{y}\right)= \\
& \left\{\begin{array}{cc}
u_{y}^{(k-1)}\left(\hat{q}_{y}\right), & q_{y}=\hat{q}_{y}, \\
u_{y}^{(k-1)}\left(\hat{q}_{y}\right)+\beta^{(k)}+2 \pi q_{y} / N_{y}, & q_{y} \neq \hat{q}_{y} .
\end{array}\right.
\end{aligned}
$$

In the functions above, $u_{x}^{(k-1)}\left(\hat{q}_{x}\right)$ and $u_{y}^{(k-1)}\left(\hat{q}_{y}\right)$ are the estimates obtained at the $(k-1)$-th iteration, $\alpha^{(k)}$ and $\beta^{(k)}$ are the $k$-th element chosen from pre-designed perturbation sequences $\mathbf{a}$ and $\mathbf{b}$ respectively. These two sequences are designed such that their elements are uniformly distributed over $[0,2 \pi / N]$. Preferably, elements are ordered in dividing this zone recursively. For example, if $N_{x}=4$, the first 4 elements in a can be set as

$$
\mathbf{a}_{4}=\{0, \pi / 4, \pi / 8,3 \pi / 8\} .
$$

The rest of elements can be a random permutation of the basic sequence $\mathbf{a}_{4}$, such that $\left(\alpha^{(k)}, \beta^{(k)}\right)$ forms various combinations. A complete search over the basic sequence requires 4 iterations and reaches a resolution of $\pi / 8$. This resolution implies an equivalent estimation error $\delta \leq \pi / 16$. Note that these are achieved for azimuth and elevation angles separately, probably not at the same time.

As we can see from Fig. $2, \delta \leq \pi / 16$ can already generate sufficiently large gap $(>25 \mathrm{~dB})$ between the right and wrong estimate for $q$. This can guarantee a high probability in obtaining an estimate for either $u_{x}$ or $u_{y}$ in the right zone after 4 iterations.
Algorithm 2 Adaptive Searching and Tracking (AST) Algorithm

S0. Initialization: Define perturbation sequence and Condition $\mathrm{A}$ and $\mathrm{B}, k=0$;

S1. Searching Stage: $k=k+1$. Let $K_{x}=N_{x}$ and $K_{y}=$ $N_{y}$, and implement the sub-algorithm in Algorithm 3;

$\mathrm{S} 2$. Check to see whether Condition $\mathrm{A}$ is satisfied. If yes, move to the tracking stage; if not, generate new scanning BF vectors using (9) and (10), and repeat the searching process in $\mathrm{S} 1$;

S3. Tracking Stage: $k=k+1$. Let $K_{x}=1$ and $K_{y}=1$, and implement the sub-algorithm in Algorithm 3;

S4. Check to see whether Condition B is satisfied. If yes, repeat S3 in the tracking stage; if not, generate new scanning BF vectors using (9) and (10), and move to the searching stage and start $\mathrm{S} 1$.

\section{Algorithm 3 Sub-algorithm in AST}

S1. At the $k$-th iteration, for every combination of $\left(q_{x}, q_{y}\right), q_{x} \in\left[0, K_{x}-1\right], q_{y} \in\left[0, K_{y}-1\right]$,

1) Apply the $\mathrm{BF}$ vector based on $w_{x}^{(k)}\left(q_{x}\right)$ and $w_{y}^{(k)}\left(q_{y}\right)$ in (9) to each subarray;

2) Combine signals from all the subarrays using the same BF vector as above, as shown in (5), and calculate the energy $\varepsilon^{(k)}\left(q_{x}, q_{y}\right)$ of the combined signal;

S2. Find $\left(\tilde{q}_{x}, \tilde{q}_{y}\right)=\max _{q_{x}, q_{y}} \varepsilon^{(k)}\left(q_{x}, q_{y}\right), q_{x} \in\left[0, K_{x}-\right.$ $1], q_{y} \in\left[0, K_{y}-1\right]$;

S3. Calculate $R_{x}^{(k)}\left(\tilde{q}_{x}, \tilde{q}_{y}\right)$ and $R_{y}^{(k)}\left(\tilde{q}_{x}, \tilde{q}_{y}\right)$ using (3), and average the cross-correlation over different iterations using a forgetting factor;

S4. Calculate $N_{x}$ possible $u_{x}^{(k)}\left(q_{x}\right)$ and $N_{y}$ possible $u_{y}^{(k)}\left(q_{y}\right)$ by applying the averaged cross-correlation to (4), $q_{x} \in$ $\left[0, N_{x}-1\right], q_{y} \in\left[0, N_{y}-1\right]$

S5. Find

$$
\begin{aligned}
& \hat{q}_{x}=\min _{q_{x} \in\left[0, N_{x}-1\right]}\left|e^{j u_{x}^{(k)}\left(q_{x}\right)}-e^{j u_{x}^{(k-1)}\left(\hat{q}_{x}\right)}\right|, \\
& \hat{q}_{y}=\min _{q_{y} \in\left[0, N_{y}-1\right]}\left|e^{j u_{y}^{(k)}\left(q_{y}\right)}-e^{j u_{y}^{(k-1)}\left(\hat{q}_{y}\right)}\right|,
\end{aligned}
$$

and obtain $u_{x}^{(k)}\left(\hat{q}_{x}\right)$ and $u_{y}^{(k)}\left(\hat{q}_{y}\right)$ as the output of the $k$-th iteration.

Remark 1: In S4 of Algorithm 2, by using the previous estimate as one of the new scanning angles, we can prevent the estimate from deviating away from a correct one in the case of a fault condition B test. If the previous one is an incorrect estimate, after entering searching stage Condition A can force the algorithm to try another set of angles with perturbation sequence included.

From the description above, we can see that using refined searching steps can limit the range of the estimation error $\delta$ and significantly reduce its impact on determining the zone for $u$. Having a reliable anchor point and using (12) to determine the estimate can largely reduce the zigzag effect. Now, a critical problem is how to design Condition $\mathrm{A}$ and $\mathrm{B}$. 


\section{B. Condition $A$ and $B$}

Condition $\mathrm{A}$ and $\mathrm{B}$ are used to control the switching between the two stages and shorten the estimation period. Condition $\mathrm{A}$ is expected to lead to a good estimate for $\arg \{R\}$, and hence a small $\delta$. Condition B is used to determine whether the current tracking is in the wrong zone and re-searching is necessary. There may be several choices for condition A and $\mathrm{B}$. Here we propose to use a threshold on the peak to average power ratio (PAPR) of the signals in each iteration for Condition A, and the degradation of total power below a threshold for Condition B.

Assume that the signal $\tilde{s}(t)$ has mean zero, and the noise in each antenna is i.i.d distributed with variance $\sigma^{2}$. The mean of signal energy for all subarrays, as represented in (5), can be derived as

$$
\begin{aligned}
& E\left\{\varepsilon^{(k)}\left(q_{x}, q_{y}\right)\right\} \\
& =\frac{\left(1-e^{j M_{x} \delta_{x}}\right)^{2}\left(1-e^{j M_{y} \delta_{y}}\right)^{2}}{\left(1-e^{j\left(\delta_{x}+2 \pi\left(q_{x}-\hat{q}_{x}\right)\right) / N_{x}}\right)^{2}\left(1-e^{j\left(\delta_{y}+2 \pi\left(q_{y}-\hat{q}_{y}\right)\right) / N_{y}}\right)^{2}} . \\
& E\left\{|\tilde{s}(t)|^{2}\right\}+N_{x} N_{y} M_{x} M_{y} \sigma^{2} .
\end{aligned}
$$

The PAPR between the maximum and averaged $E\left\{\varepsilon^{(k)}\left(q_{x}, q_{y}\right)\right\}$ over all combinations of $\left(q_{x}, q_{y}\right)$, where the maximum is obtained at $q=\hat{q}$, is given by

$$
\rho=\frac{E\left\{\varepsilon^{(k)}\left(\hat{q}_{x}, \hat{q}_{y}\right)\right\}}{\frac{1}{N_{x} N_{y}} \sum_{q_{x}, q_{y}} E\left\{\varepsilon^{(k)}\left(q_{x}, q_{y}\right)\right\}} .
$$

Using the same parameters as in Fig. 2, Fig. 3 demonstrates the ratio for various estimation errors $\delta_{x}=\delta_{y}$, at several values of $\gamma \triangleq E\left\{|\tilde{s}(t)|^{2}\right\} / \sigma^{2}$ which denotes the received SNR at each antenna. Based on the figure, one can choose a threshold $\rho_{t h}$ corresponding to the desired accuracy of $\delta_{x}$ for a given SNR. Condition A can thus be defined as $\rho>\rho_{t h}$. When Condition A is satisfied, there is a high probability that the estimation error $\delta$ is smaller than the targeted accuracy. One shortcoming is that such a threshold is dependent of the SNR, while its estimation is an on-going research problem.

For Condition B, we use the peak energy obtained when Condition $\mathrm{A}$ is satisfied as a base point. Condition $\mathrm{B}$ is thus defined as the instant energy in each iteration in the tracking stage is smaller than a pre-set percent $c$ (e.g., $c=40 \%)$ of this base point.

As will be seen from the simulation results in Section V, the proposed AST algorithm works well through the use of the two conditions. We will also demonstrate the impact of using different PAPR thresholds and the percentage values on estimation performance there. Nevertheless, their optimization is yet to be studied.

\section{Training Sequence Design}

Similar to the DBS algorithm in [7], the proposed algorithm can also work in a blind way without requiring the knowledge of received signals. However, a long operating period is required to ensure that the actual average power of $|\tilde{s}(t)|^{2}$ are similar for different subframes. Instead, we can design special training sequences to speed up this process.

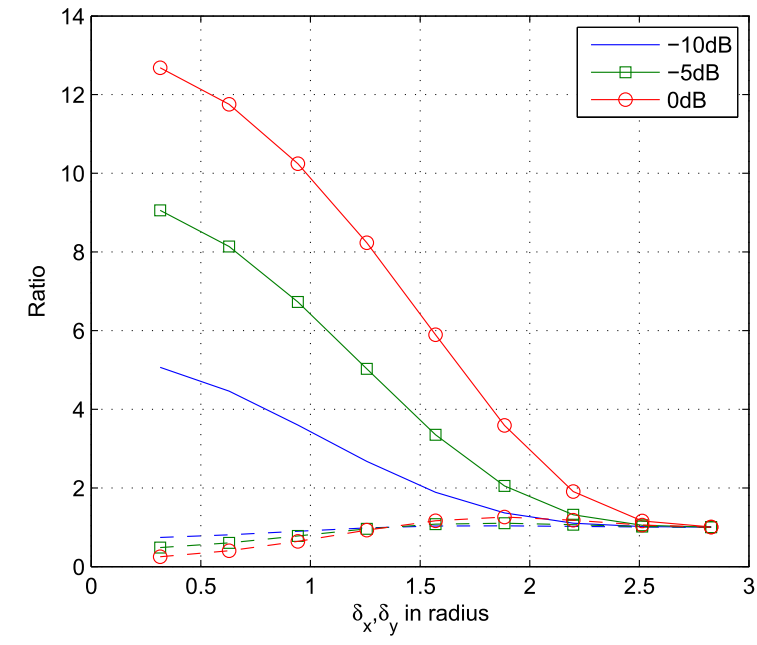

Fig. 3. The ratio $\rho$ for various $\delta_{x}=\delta_{y}$ at per-antenna SNR $-10,-5$ and 0 $\mathrm{dB}$. Solid curves for the PAPR ratios, and dashed curves for the ratio between the second largest and the average.

One important property of the training sequence is that each subframe should have the same power, even when the receiver is not synchronized to the transmitted signal. We can design such sequences by generating a basic sequence of the subframe length, then repeating the basic sequence and multiplying each basic sequence by one element from a $\{1,-1\}$ pseudo-random sequence. Multiplying by the pseudo-random sequence can eliminate the spectrum line due to signal repetition.

\section{Simulation Results}

In this section, we present simulation results for the proposed algorithms. We consider a square array with $2 \times 2$ subarrays, and each subarray has $4 \times 4$ antenna elements. The space between adjacent antennas is uniform and equals to $\lambda / 2$. The AoA of incoming signals is uniformly distributed on $[-\pi / 4, \pi / 4]$, in both elevation and azimuth direction. The forgetting factor in averaging the cross-correlation in all the algorithms are 0.4 . The length of every subframe is 1 . The simulation results are averaged over 4000 trials, and the number of maximum iterations in each trial is set as 50 . The threshold in Condition A is set as 3, 3.5, 4 and 4.5 for perantenna SNR $\gamma=-11,-9,-7$, and $-5 \mathrm{~dB}$ respectively, unless noted otherwise.

Fig. 4 compares the estimation performance for AST, DBS1 and the original DBS algorithms. The mean square error (MSE) between the actual $u_{x}$ and $u_{y}$ and their estimates is plotted against the averaged numbers of subframes spent in obtaining the estimates. The number of subframes in each iteration is fixed as 16 in DBS and DBS-1, while it is 16 in the searching stage and 1 in the tracking stage in AST. Hence it is averaged over different trials for AST in the plot. From the figure we can see that AST initially uses more subframes to find better initial estimates of $\arg \{R\}$, and then quickly outperforms DBS-1. Unlike the error floor in DBS-1 and DBS, which is caused by the estimation error in $\arg \{R\}$ and the phase discontinuity problem discussed before, the 


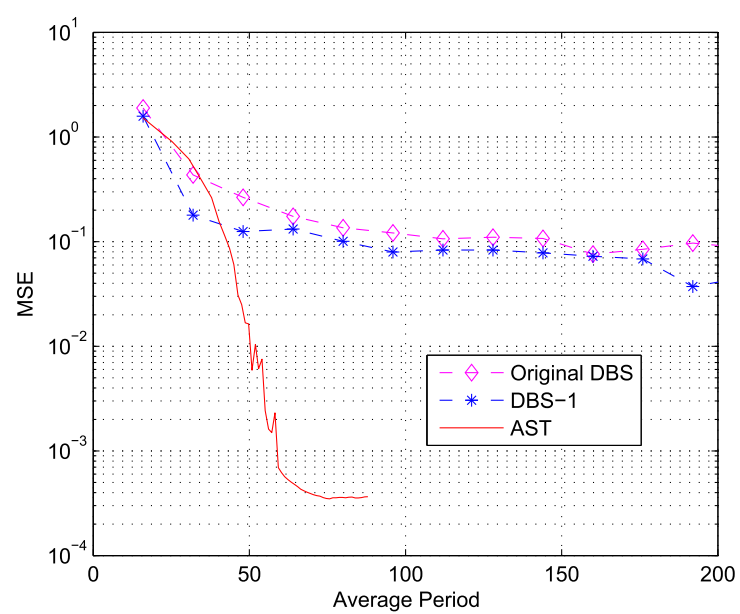

Fig. 4. MSE at averaged estimation period for AST, DBS-1 and DBS, when $\mathrm{SNR}=-5 \mathrm{~dB}$.

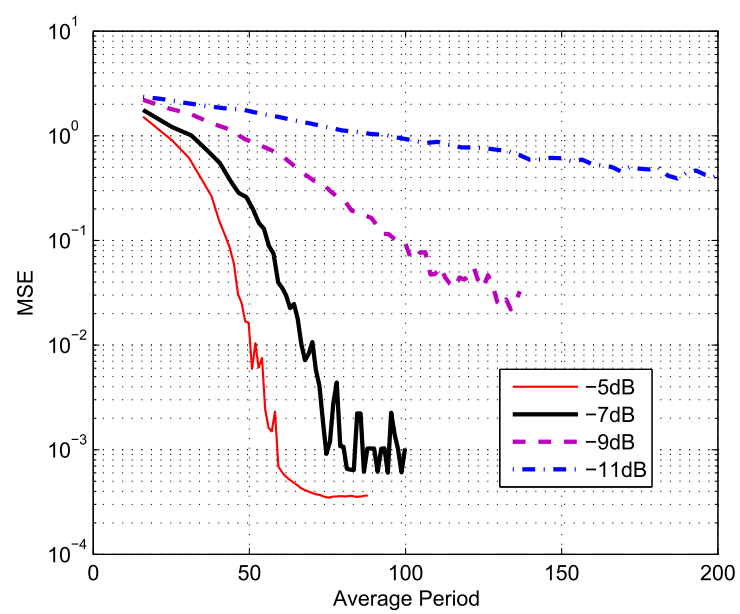

Fig. 5. MSE at different SNRs for AST, when $c=0.3$.

MSE of AST decreases quickly with the number of iterations increasing. The small error floor below $10^{-3}$ for AST is likely caused by the large forgetting factor.

Fig. 5 shows the MSE at different SNRs for the proposed AST algorithm. Although the estimation performance degarades quickly with SNR decreasing, larger error floor clearly does not exist. Averaged shifting times from tracking to searching stages is $0.21,0.69,2.43$ and 6.67 for SNR -5 , $-7,-9$ and $-11 \mathrm{~dB}$.

Fig. 6 demonstrates the impact of the parameters $\rho_{t h}$ and $c$ in the two conditions on the estimation performance. The performance variation is small according to the figure, which shows the robustness of the AST scheme to a certain range of parameters.

\section{CONCLUSIONS}

In this paper, we investigated and proposed novel AoA estimation algorithms for localized hybrid arrays. We first

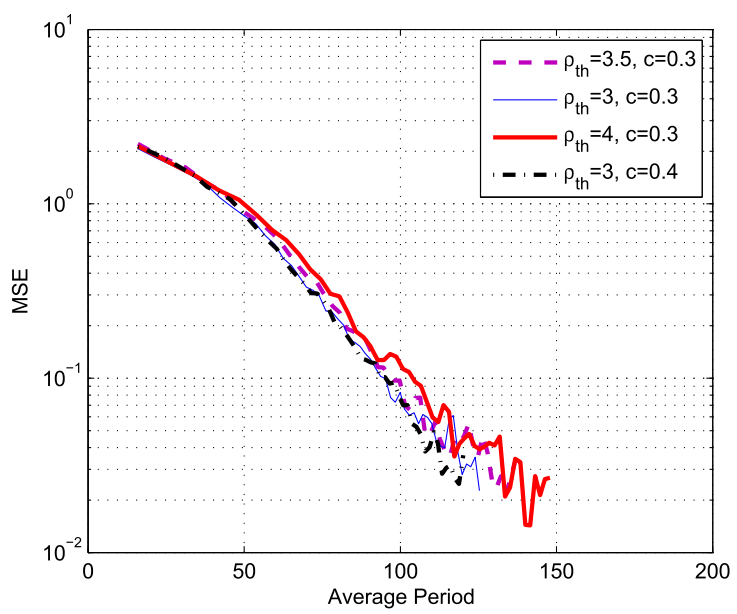

Fig. 6. MSE for various configurations of $\rho_{t h}$ and $c$ in AST, where $\mathrm{SNR}=-9$ $\mathrm{dB}$.

analysed the limitations of existing and improved DBS algorithms for AoA estimation, and then proposed an adaptive searching and tracking (AST) algorithm to overcome these limitations and speed up the estimation. The AST algorithm uses carefully designed updating functions for propagating estimates between different subarrays and iterations, and uses two conditions to automatically switch between searching and tracking stages. Simulation results show that the proposed AST algorithm can significantly reduce the estimation period and improve the estimation performance.

\section{REFERENCES}

[1] T. Rappaport, S. Sun, R. Mayzus, H. Zhao, Y. Azar, K. Wang, G. Wong J. Schulz, M. Samimi, and F. Gutierrez, "Millimeter wave mobile communications for $5 \mathrm{~g}$ cellular: It will work!" Access, IEEE, vol. 1, pp. 335-349, 2013.

[2] S. Costanzo, I. Venneri, G. D. Massa, and G. Amendola, "Hybrid array antenna for broadband millimeter-wave applications," Progress In Electromagnetics Research, vol. 83, pp. 173-183, 2008.

[3] Y. J. Guo, X. Huang, and V. Dyadyuk, "A hybrid adaptive antenna array for long-range mm-wave communications," IEEE Antennas and Propagation Magazine, vol. 54 (2), pp. 271-282, April 2012.

[4] O. E. Ayach, S. Rajagopal, S. Abu-Surra, Z. Pi, and R. W. H. Jr., "Spatially sparse precoding in millimeter wave mimo systems," IEEE Transactions on Wireless Communications, vol. 13, no. 3, pp. 1499-, 2014.

[5] P. Liao and R. York, "A new phase-shifterless beam-scanning technique using arrays of coupled oscillators," Microwave Theory and Techniques, IEEE Transactions on, vol. 41, no. 10, pp. 1810-1815, Oct 1993.

[6] P. Stoica and A. Nehorai, "MUSIC, maximum likelihood, and cramer-rao bound: further results and comparisons," Acoustics, Speech and Signal Processing, IEEE Transactions on, vol. 38, no. 12, pp. 2140-2150, Dec 1990.

[7] X. Huang, Y. Guo, and J. Bunton, "A hybrid adaptive antenna array," Wireless Communications, IEEE Transactions on, vol. 9, no. 5, pp. 17701779, May 2010.

[8] X. Huang and Y. Guo, "Frequency-domain AoA estimation and beamforming with wideband hybrid arrays," Wireless Communications, IEEE Transactions on, vol. 10, no. 8, pp. 2543-2553, August 2011.

[9] A. Alkhateeb, O. El Ayach, G. Leus, and R. Heath, "Channel estimation and hybrid precoding for millimeter wave cellular systems," Selected Topics in Signal Processing, IEEE Journal of, vol. 8, no. 5, pp. 831846, Oct 2014. 